\title{
Management of Chemotherapy-Induced Nausea and Vomiting with Trastuzumab Deruxtecan: A Case Series
}

\author{
Matthew Stankowicz ${ }^{\mathrm{a}}$ Lauren Mauro ${ }^{\mathrm{b}}$ Kathleen Harnden ${ }^{\mathrm{b}} \quad$ Angela Pennisi $^{\mathrm{b}}$ \\ ${ }^{a}$ Department of Pharmacy, Inova Schar Cancer Institute, Fairfax, VA, USA; ${ }^{b}$ Breast Medical Oncology, Inova Schar \\ Cancer Institute, Fairfax, VA, USA
}

\section{Established Facts}

- Trastuzumab deruxtecan is a first in-class human epidermal growth factor receptor 2 (HER2)-targeted monoclonal antibody linked to an SN-38 moiety.

- Food and Drug Administration approval for metastatic HER2-positive breast cancer in patients who have failed at least two previous therapies.

- Black box warnings for interstitial lung disease/pneumonitis and embryo-fetal toxicity.

\section{Novel Insights}

- Rates of chemotherapy-induced nausea and vomiting (CINV) of $80 \%$ in clinical trials with no recommendations for management in package insert.

- No standardized emetogenic protocols in clinical trials, making real-world practice recommendations difficult.

- Management based on moderate emetic potential resulted in a $28.9 \%$ rate of CINV.

\section{Keywords}

Breast neoplasms · Drug therapy · Evidence-based pharmacy practice

\begin{abstract}
Introduction: Trastuzumab deruxtecan is a monoclonal antibody linked to a chemotherapy moiety that was recently approved by the Food and Drug Administration (FDA) for the treatment of metastatic human epidermal growth factor receptor 2 (HER2) positive breast cancers. There are labeled black box warnings for interstitial lung disease (ILD)/pneumonitis and embryo-fetal toxicity. Additionally, chemotherapy-induced nausea and vomiting (CINV) was reported to be as high as $78 \%$ ( $~ 8 \%$ grade 3 or higher) in phase I and II clin-
\end{abstract}

ical trials. Clinical trial and package insert recommendations for the management of CINV are not available, making realworld management difficult. Case Presentation: We reviewed the first 10 patients who received trastuzumab deruxtecan at our hospital-based community cancer center to determine if CINV management was adequate. We found a rate of $28.9 \%$ CINV (all grade 1 and 2 ) despite treatment as a moderate emetic potential regimen. Interventions by the treatment team to manage trastuzumab deruxtecan as a high-risk emetic regimen resulted in reduced CINV and ongoing treatment for all patients. Discussion and Conclusion: This review indicates that management of CINV for patients receiving trastuzumab deruxtecan should follow recommendations for regimens with a high-risk emetic potential.

c) 2020 S. Karger AG, Basel 


\section{Introduction}

Trastuzumab deruxtecan is a monoclonal antibody targeting the human epidermal growth factor receptor 2 (HER2) linked to a topoisomerase inhibitor payload. The drug-to-antibody ratio is significantly higher than the drug-to-antibody ratio for other conjugated antibody complexes, such as trastuzumab emtansine. Additionally, the topoisomerase inhibitor payload has a shorter halflife than most chemotherapeutic agents, and is a unique mechanism of action that most breast cancer patients have not previously received. These unique molecular characteristics, along with a specific cleavable linker are thought to increase tumor cell death while limiting systemic toxicities [1]. Currently, the US Food and Drug Administration (FDA) has approved trastuzumab deruxtecan for the treatment of HER2(+) metastatic breast cancer in patients who have failed at least two previous anti-HER2 therapies in the metastatic setting [2]. Trastuzumab deruxtecan is labeled with black box warnings for interstitial lung disease (ILD)/pneumonitis and embryofetal toxicity.

The black box warning for ILD/pneumonitis is due to $9 \%$ of 234 patients developing ILD/pneumonitis, with $2.6 \%$ of the patients treated developing grade 5 (fatal) ILD/pneumonitis across both phase I and phase II clinical trials $[1,3]$. Due to this documented risk of ILD/pneumonitis, our facility implemented a monitoring plan for ILD that required baseline pulmonary function tests alternating every 6 weeks with computerized tomography of the chest. Additional adverse event monitoring was planned according to package insert recommendations, including: neutropenia (use of colony-stimulating factors), left ventricular dysfunction (ejection fraction at baseline and at regular intervals), and embryo-fetal toxicity (active beta-HCG testing in pre-menopausal women who are still sexually active, as well as patient education on contraception).

Despite clinical trials reporting near $80 \%$ all-grade chemotherapy-induced nausea and vomiting (CINV), the package insert does not provide specific recommendations for the management of CINV [1-3]. This is likely due to the DESTINY-Breast01 trial allowing use of premedications at the discretion of the investigator based on local standards, with a rate of $\sim 10 \%$ for grade 3 and 4 CINV. Due to the inconsistent reporting of CINV premedications administered in the DESTINY-Breast01 trial, and unclear recommendations in the package insert, we were left to assume the best approach to CINV prevention in our patients.

Because the clinical trials and package insert did not provide recommendations for CINV management, we made the decision to pre-medicate with standard moderate-risk emetic pre-medications, per National Compre-
Table 1. Select baseline demographics

\begin{tabular}{lc} 
Age, years & $52.1(38-80)$ \\
ER/PR(+) & $3(30)$ \\
No. of regimens for metastatic disease & $3.7(2-7)$ \\
Site of metastases & \\
$\quad$ Brain & 3 \\
$\quad$ Bone, lung & 1 \\
$\quad$ Lung & 1 \\
$\quad$ Bone, brain, lungs & 1 \\
$\quad$ Liver, lung & 1 \\
Chest wall & 1 \\
$\quad$ Brain, lung & 1 \\
Bone & 1 \\
No. of doses & $4.5(3-6)$ \\
Dose, mg & $392(300-490)$ \\
\hline
\end{tabular}

Values are expressed as median (range), number, or number (percentage).

hensive Cancer Center Network (NCCN) guideline recommendations [4]. We conducted a retrospective review of CINV in the first 10 patients who received trastuzumab deruxtecan at our outpatient facilities and formulated a recommendation for CINV prevention regimens in all patients who receive trastuzumab deruxtecan.

\section{Case Report and Case Presentation}

Prior to addition to our formulary, trastuzumab deruxtecan was used in 10 patients across our hospital-based outpatient community cancer center. Their charts were reviewed to assess baseline demographics, as well as CINV graded according to the Common Terminology Criteria for Adverse Events (CTCAE) version 5.0, where grade 0 is absence of toxicity and grade 5 is death [5]. Additionally, we evaluated pharmacologic interventions made to correct CINV, including adjustment to pre-medications, adjustment to home medication regimens, or addition of home or infusion pre-medications. Finally, we assessed the pre-medication regimen (as applicable to CINV) to determine if there is an ideal combination of pre-medications to decrease CINV in patients receiving trastuzumab deruxtecan.

The first 10 patients (average age 52.1 years) treated at our facility received a total of 45 doses of trastuzumab deruxtecan. Select baseline characteristics are described in Table 1. The average number of previous therapies at the time of first-dose administration was 3.7 (including chemotherapy, radiation therapy, and targeted therapy). Of note, only 3 patients had estrogen receptor (ER)- or progesterone receptor (PR)- positive tumors.

Of the 45 doses administered, patients had a documented toxicity attributed to $22(48.9 \%)$ of those doses with 13 doses $(28.9 \%)$ leading to reported CINV. Five patients reported grade $1 \mathrm{CINV}$ after 5 unique doses while 5 patients reported grade 2 CINV after a total of 8 unique doses. Importantly, no patients reported grade 2 CINV after previously reporting grade $1 \mathrm{CINV}$; however, 2 patients did report only a grade $1 \mathrm{CINV}$ after reporting grade $2 \mathrm{CINV}$ with the previous dose. Descriptions of the pre-medications and clinical interventions for the patients who developed CINV are outlined in Table 2. 
Table 2. Pre-medications and clinical interventions for patients who developed CINV

\begin{tabular}{ll}
\hline Pre-medication regimen & Clinical intervention \\
\hline Grade 1 CINV & None \\
Dex $12 \mathrm{mg}$ PO, Ond $16 \mathrm{mg}$ PO & None \\
Dex $12 \mathrm{mg}$ PO, Ond $16 \mathrm{mg}$ PO & None \\
Dex $8 \mathrm{mg}$ IV, Ond $16 \mathrm{mg}$ IV & None \\
Dex $8 \mathrm{mg}$ PO, Ond $16 \mathrm{mg}$ PO & None \\
Dex $12 \mathrm{mg}$ IV, Dex $16 \mathrm{mg}$ IV, Diph $25 \mathrm{mg}$ IV & \\
Grade 2 CINV & Increase home medication \\
Dex $12 \mathrm{mg}$ IV, Ond $16 \mathrm{mg}$ IV & Add home medication \\
& Add home medication (2) \\
Dex $12 \mathrm{mg}$ PO, Ond $16 \mathrm{mg}$ PO & Increase home medication \\
Dex $8 \mathrm{mg}$ IV, Ond $16 \mathrm{mg}$ IV & Increase home medication \\
Dex $12 \mathrm{mg}$ PO, Ond $16 \mathrm{mg}$ PO, Diph $12.5 \mathrm{mg}$ IV & Increase home medication \\
Dex $12 \mathrm{mg}$ PO, Ond $16 \mathrm{mg}$ PO & Add Home medication \\
& Add IV pre-medication \\
Dex $12 \mathrm{mg}$ PO, Ond $16 \mathrm{mg}$ PO & Switch all to IV pre-medications \\
\hline CINV, chemotherapy-induced nausea and vomiting; Dex, dexamethasone; Diph, diphenhydramine; IV, in- \\
\hline
\end{tabular}

Importantly, no patients developed ILD/pneumonitis during treatment with trastuzumab deruxtecan. One patient had respiratory symptoms but tested positive for influenza type $\mathrm{A}$, which was determined to be unrelated to treatment. Additional toxicities reported include fatigue $(n=3$ doses), blurry vision $(n=2)$, cough $(n=2)$, mucositis $(n=2)$, nose bleed $(n=2)$, pain $(n=2)$, rash $(n=2)$, decreased appetite $(n=1)$, diarrhea $(n=1)$, edema $(n=1)$, gastritis $(n=1)$, infusion reaction $(n=1)$, and neutropenia $(n=1)$. No toxicities were considered grade 3 or higher based on our chart review.

\section{Discussion and Conclusion}

The NCCN guidelines on antiemesis (version 2.2020) recently added trastuzumab deruxtecan to the list of "moderate emetic risk parenteral anticancer agents" [4]. Per these guidelines, which we use as our institutional standards, this would require ondansetron 16-24 mg PO (by mouth) with dexamethasone $12 \mathrm{mg}$ IV (intravenous) or PO as a pre-medication with either ondansetron or dexamethasone continued as a home medication [4]. With the exception of 3 administered doses in our first 10 patients, all of the pre-medications and take-home medications were in compliance with these recommendations, yet we still had a rate of CINV of $28.9 \%$. Of those three doses administered without the recommended antiemetic regimen, all doses were complicated by CINV, two grade 2 and one grade 1 .

Our review found lower rates of CINV than in clinical trials $[1,3]$. One such factor that may explain this discrepancy is the use of pre-medications. While based on institutional standards in the clinical trials, there is no publicly available information on the pre-medications used. The rates reported are consistent with a moderate-risk emetogenic potential therapy (30-90\%) based on the classification by the NCCN guidelines [4] and Hesketh et al. [6]; however, without knowledge of the pre-medications used, this may be an underestimation of the risk of CINV. This is highlighted in our retrospective review, wherein nearly one-third of our doses lead to a report of CINV despite adequate pre-medications for moderate-risk emetogenicity.

When this trend of increased rates of CINV was noticed in these patients, the decision was made to treat trastuzumab deruxtecan as a high-risk emetogenic regimen. As such, we have made the institutional decision to pre-medicate with aprepitant $130 \mathrm{mg} I \mathrm{~V}$, dexamethasone $12 \mathrm{mg}$ IV, and ondansetron $16 \mathrm{mg}$ IV. Additionally, takehome prescriptions include dexamethasone, ondansetron, and olanzapine per the NCCN guideline recommendations [4]. Since implementing these changes, we have administered 3 doses of fam-trastuzumab to 2 unique patients, without any reported CINV.

There are inherent limitations to this report, including the retrospective nature and small sample size; however, there is a trend towards classifying trastuzumab deruxtecan as a high-risk emetic agent. This is especially important in patients receiving this medication for the current indication as they are heavily pre-treated and discontinuation due to CINV may leave the patient without further treatment options. Additionally, with anticipated FDA approvals in HER2(+) non-small lung cancer and gastric cancer, it is imperative that CINV is effectively addressed in preparation for many more patients to be receiving this regimen. 
As a result of this review, our institution will manage all patients receiving trastuzumab deruxtecan according to recommendations for high-risk emetic potential, and we urge other practitioners to do the same.

\section{Acknowledgement}

The authors would like to acknowledge the Inova Schar Cancer Institute Department of Breast Medical Oncology for providing assistance in evaluating patient outcomes and providing continued care to these patients.

\section{Conflict of Interest Statement}

The authors have no conflicts of interest to declare.

\section{Statement of Ethics}

This was determined to be a quality improvement initiative and did not require review by the institutional review board. All patient information was de-identified during data collection and reporting.

\section{Funding Sources}

This study did not use external funding sources.

\section{Author Contributions}

All authors contributed to drafting and editing the manuscript, as well as evaluating patient charts and providing clinical care.

\section{References}

1 Tamura K, Tsurutani J, Takahashi S, Iwata H, Krop IE, Redfern C, et al. Trastuzumab deruxtecan (DS-8201a) in patients with advanced HER2-positive breast cancer previously treated with trastuzumab emtansine: a dose-expansion, phase 1 study. Lancet Oncol. 2019 Jun;20(6):816-26.

2 Enhertu [package insert]. Basking Ridge (NJ): Daiichi Sankyo, Inc.; 2019.
3 Modi S, Saura C, Yamashita T, Park YH, Kim SB, Tamura K, et al.; DESTINYBreast01 Investigators. Trastuzumab deruxtecan in previously treated HER2-positive breast cancer. N Engl J Med. 2020 Feb; 382(7):610-21.

4 National Comprehensive Cancer Network. Antiemesis version 2.2020 [internet]. [Updated 2020 Apr; cited 2020 June 18]. Available from: https://www.nccn.org/ professionals/physician_gls/pdf/antiemesis.pdf.
5 U.S. Department of Health and Human Services. Common Terminology Criteria for Adverse Events (CTCAE) version 5.0 [internet]. [Updated 2017 Nov; cited 2020 June 18]. Available from: https://ctep.cancer.gov/protocoldevelopment/electronic_applications/ docs/ctcae_v5_quick_reference_8.5x11.pdf.

6 Hesketh PJ, Kris MG, Grunberg SM, Beck T, Hainsworth JD, Harker G, et al. Proposal for classifying the acute emetogenicity of cancer chemotherapy. J Clin Oncol. 1997 Jan;15(1): 103-9. 